\title{
Spectrin regulates Hippo signaling by modulating cortical actomyosin activity
}

\author{
Hua Deng, Wei Wang, Jianzhong Yu, Yonggang Zheng, Yun Qing, Duojia Pan* \\ Department of Molecular Biology and Genetics, Howard Hughes Medical Institute, \\ Johns Hopkins University School of Medicine, Baltimore, United States
}

\begin{abstract}
The Hippo pathway controls tissue growth through a core kinase cascade that impinges on the transcription of growth-regulatory genes. Understanding how this pathway is regulated in development remains a major challenge. Recent studies suggested that Hippo signaling can be modulated by cytoskeletal tension through a Rok-myosin II pathway. How cytoskeletal tension is regulated or its relationship to the other known upstream regulators of the Hippo pathway remains poorly defined. In this study, we identify spectrin, a contractile protein at the cytoskeleton-membrane interface, as an upstream regulator of the Hippo signaling pathway. We show that, in contrast to canonical upstream regulators such as Crumbs, Kibra, Expanded, and Merlin, spectrin regulates Hippo signaling in a distinct way by modulating cortical actomyosin activity through non-muscle myosin II. These results uncover an essential mediator of Hippo signaling by cytoskeleton tension, providing a new entry point to dissecting how mechanical signals regulate Hippo signaling in living tissues. DOI: 10.7554/eLife.06567.001
\end{abstract}

*For correspondence: djpan@ jhmi.edu

\section{Competing interests:}

See page 14

Funding: See page 14

Received: 19 January 2015 Accepted: 30 March 2015 Published: 31 March 2015

Reviewing editor: Janet Rossant, University of Toronto, Canada

Copyright Deng et al. This article is distributed under the terms of the Creative Commons Attribution License, which permits unrestricted use and redistribution provided that the original author and source are credited.

\section{Introduction}

The Hippo signaling pathway controls organ size in Drosophila through coordinated regulation of cell growth, proliferation, and apoptosis (Harvey and Tapon, 2007; Pan, 2007; Halder and Johnson, 2011). This pathway involves a core kinase cascade in which the Hippo-Salvador (Hpo-Sav) kinase complex phosphorylates and activates the Warts-Mats (Wts-Mats) kinase complex, which in turn inactivates the Yorkie (Yki) oncoprotein through phosphorylation. This phosphorylation event excludes Yki from the nucleus, where it normally functions as a coactivator for the expression of Hippo pathway target genes. The conserved function of Hippo signaling in mammalian growth control and tumorigenesis has stimulated much interest in understanding the regulation of this pathway in development, regeneration, and disease (Zhao et al., 2008; Pan, 2010; Barry and Camargo, 2013; Harvey et al., 2013; Johnson and Halder, 2014).

Genetic studies in Drosophila suggest that the Hippo kinase cascade is modulated by a diverse array of upstream regulators (Boggiano and Fehon, 2012; Enderle and McNeill, 2013). Prominent among these are three membrane-associated tumor suppressor proteins, Expanded (Ex), Merlin (Mer) and Kibra, which act semi-redundantly to activate downstream signaling, by recruiting the core kinase cassette to the plasma membrane or cytoplasmic sequestration of Yki through direct binding. Other tumor suppressor proteins implicated as upstream regulators of the Hippo kinase cascade include the atypical cadherins Fat (Ft) and Dachsous (Ds), apical basal polarity regulators Crumbs (Crb), Scribble (Scrib), Discs large (Dlg), and Lethal giant larvae (Lgl), the Ste20-like kinase Tao-1, the protein tyrosine phosphatase Pez, and the cell adhesion molecule Echinoid (Ed). At least some of these tumor suppressors have been shown to play a conserved role in Hippo signaling in mammals, which have also acquired additional regulators such as Angiomotin (Amot), $\alpha$-catenin, and G protein-coupled receptors (GPCRs) (Yu and Guan, 2013).

In an exciting recent development, studies in cultured mammalian cells have implicated YAP and $T A Z$, the mammalian counterpart of $Y k i$, as key mediators of mechanotransduction, whereby changes 
eLife digest Organs including the liver, eyes, and lungs are made up of millions of cells, and how these organs stop growing once they reach their final size has fascinated scientists for decades. The cells in developing organs must communicate with each other and respond appropriately to the signals that they receive from other cells. This requires so-called "signaling pathways". One such pathway that involves a protein called Hippo is known to control when cells should grow and divide and when they should stop. If this pathway does not work correctly, it can cause too many cells to be formed, which may result in cancer.

The Hippo signaling pathway can also be regulated by an extensive network of protein filaments found within cells, called the cytoskeleton. This network can exert forces on the cells, which can have a major impact on cell growth. However, the mechanism behind the interaction between the cytoskeleton and the Hippo signaling pathway is poorly understood.

Now, Deng et al. have engineered fruit flies in which the expression of individual genes had been artificially reduced, and looked for flies that had enlarged wings. Three genes identified in these experiments encode different subunits of a large spring-like protein, called spectrin, which is part of the cytoskeleton. This suggests that normally spectrin limits wing size. Furthermore, spectrin was also found to control the size of other organs in the fruit flies, such as the eyes and ovaries. In all of these organs, the Hippo signaling pathway failed to work properly in the absence of spectrin. Deng et al. then further explored the relationship between spectrin and Hippo signaling and found that cells without spectrin show abnormally high levels of tension in their cytoskeleton. When flies that lacked spectrin were engineered to reduce this tension, these flies developed normal sized organs. These findings reveal the importance of cytoskeleton tension in controlling tissue growth, and provide a new entry point to understand how normal tissues grow to their characteristic size and how such process goes awry in cancer.

DOI: 10.7554/eLife.06567.002

in cell-extracellular matrix (ECM) interaction, cell shape, or the actomyosin cytoskeleton influence cellular behaviors such as proliferation and differentiation (Dupont et alo, 2011; Wada et al., 2011; Aragona et al., 2013). Molecular interrogation of this mechanotransduction process suggests that the subcellular localization and thus the activity of YAP/TAZ is regulated by the contractile actomyosin through a Rok (Rho-associated protein kinase)-myosin II pathway. In Drosophila, excessive actin polymerization or activation of Rok-myosin II also lead to increased Yki activity (Fernandez et al., 2011; Sansores-Garcia et al., 2011; Rauskolb et al., 2014), suggesting that cytoskeleton tension is a conserved regulator of Hippo signaling in diverse animals. Despite these exciting progresses, important questions remain: what is the relationship between the actomyosin cytoskeleton and the other reported upstream regulators such as Kibra, Ex, and Mer in Hippo pathway regulation? Furthermore, since mechanical force can be sensed and transduced in many subcellular regions such as cell-cell junction, plasma membraneassociated cytoskeleton cortex, cytoplasmic stress fiber and even nuclear membrane-associated cortex, what is the exact nature of the cytoskeletal force that is relevant to Hippo signaling?

Spectrin is a large spring-like protein that forms the spectrin-based membrane skeleton (SBMS) right beneath the plasma membrane by crosslinking short F-actin and binding integral membrane proteins (Bennett and Baines, 2001). Spectrin proteins are conserved in all eukaryotes from protozoa to humans (Baines, 2009). Spectrin exists mainly as heterotetramers of $\alpha$ and $\beta$ subunits, in which the $\alpha$ and $\beta$ subunits are assembled side to side in an antiparallel fashion to form rod-like $\alpha \beta$ dimers that in turn self-associate head to head to form tetramers. With the help of Adducin protein, these tetramers crosslink with short F-actin to form a lattice-like network that supports the structural stability of the plasma membrane. Indeed, spectrin was first identified as proteins that contribute to the mechanical resilience of erythrocytes (Bennett and Gilligan, 1993), and mutations in spectrin genes result in severe anemia characterized by abnormally shaped erythrocytes with increased membrane fragility (Delaunay, 2007). A recent study reported that the SBMS functions independently of actin dynamics to maintain the pre-stress status of touch receptor neurons (TRNs) in Caenorhabditis elegans and therefore enhances the overall mechanosensitivity of these neurons (Krieg et alı, 2014). Whether the SBMS plays a direct role in mechanotransduction, or the relationship between the SBMS and the actomyosin cytoskeleton in mechanotransduction, is less clear. 
The fruit fly Drosophila encodes one $\alpha$ subunit $(\alpha-S p e c)$ and two $\beta$ subunits ( $\beta$-Spec and $\beta$ Heavy-spec or $\beta \mathrm{H}$-Spec), which generate two spectrin tetramers, $(\alpha \beta)_{2}$ and $(\alpha \beta \mathrm{H})_{2}$. In Drosophila ovarian follicle cells, $\beta-\mathrm{Spec}$ and $\beta \mathrm{H}-\mathrm{Spec}$ are localized to the basolateral and apical membrane, respectively, while $\alpha$-Spec is localized along the entire apical-basal axis (Lee et al., 1997). Here, we report the identification of spectrin genes as negative growth regulators and upstream regulators of the Hippo signaling pathway in Drosophila. Interestingly, unlike the previously reported upstream regulators of the Hippo pathway such as Crumbs, Kibra, Ex and Mer, spectrin regulates Hippo signaling through a distinct mechanism by modulating the activity of nonmuscle myosin II. These results uncover an essential mediator of Hippo signaling by cytoskeleton tension at the membrane-cytoskeleton interface, providing a new entry point to dissecting how mechanical signals regulate Hippo signaling in living tissues.

\section{Results}

In a genome-wide RNAi screen, we identified $\alpha$-spec, $\beta$-spec, and $\beta \mathrm{H}$-spec as tumor suppressors based on the enlarged wing phenotype produced by Gal4-mediated overexpression of UAS-RNAi transgenes in the wing tissue (Figure 1A-B). Antibody staining confirmed that the RNAi transgenes of $\alpha$-spec and $\beta H$-spec efficiently knocked down the expression of the respective genes in the imaginal discs (Figure 1-figure supplement 1). Furthermore, consistent with previous studies in ovarian follicle cells (Lee et al., 1997), $\beta \mathrm{H}$-Spec is mainly localized to the apical membrane of the imaginal disc epithelial cells (Figure 1-figure supplement $1 E-E^{\prime \prime}$ ), while $\alpha$-Spec localizes to both lateral and apical domains in these cells (Figure 1-figure supplement 1B-B'). Given that RNAi knockdown of any of the three spectrin genes produced a similar phenotype and that $\alpha$-Spec is the major component of both apical and lateral SBMS in the epithelial cells (Lee et al., 1997), we focused our analysis on $\alpha$-spec unless otherwise indicated.

The wing overgrowth phenotype resulting from $\alpha$-spec RNAi was suppressed by Wts overexpression (Figure 1A-B), suggesting a potential relationship between the SBMS and Hippo signaling. To explore this relationship further, we tested whether spectrin knockdown enhances the mild overgrowth phenotype caused by mutations in upstream regulators of the Hippo signaling, considering that they usually work redundantly to regulate Hippo signaling (Hamaratoglu et alo, 2006). Reducing $\alpha$-spec expression in ex $\mathrm{x}^{\mathrm{e}}$ mutant cells significantly enhanced the overgrowth phenotype caused by $\mathrm{ex}^{\mathrm{e}}$ in both the adult notum and eye tissue (Figure 1C-D). Another hallmark of defective Hippo signaling is the alteration of interommatidial cell number in pupal retina. Wild-type eyes have an average of 12 interommatidial cells surrounding each unit eye (Carthew, 2007) (Figure 1E). Mutants of upstream regulators of the Hippo pathway display a mild increase in interommatidial cells, with loss of ex, mer, and kibra resulting in 1.3, 4.2, and 3.8 extra cells per cluster (ECPC), respectively (Figure 1E-L). Pupal retina with $\alpha$-spec RNAi had an average of 2.4 ECPC (Figure 1F), which is stronger than ex, but is milder than mer or kibra mutants. Interestingly, combinations of $\alpha$-spec RNAi with any of these mutations resulted in a striking synergistic phenotype, with $\alpha$-specRNAi;ex, $\alpha$-specRNAi;mer, and $\alpha$-specRNAi; kibra double mutant retina producing 17.7, 24.5 and 10.3 ECPC, respectively (Figure 1E-L). Like $\alpha$-spec, RNAi of $\beta$-spec or $\beta H$-spec also led to a mild increase of interommatidial cells (Figure 1-figure supplement $\left.2 A-B^{\prime \prime}\right)$, which were confirmed in mutant clones for a null allele of $\alpha$-spec $\left(\alpha-\operatorname{spec}^{\text {rg41 }}\right)$ or $\beta$-spec $\left(\beta\right.$-spec $\left.{ }^{9}\right)$ (Figure 1-figure supplement 2C- $D^{\prime \prime}$ ). Taken together, these results suggest that spectrin functions in conjunction with the known upstream regulators of the Hippo pathway to regulate tissue growth.

To further corroborate our hypothesis that spectrin is an upstream regulator of the Hippo signaling, we examined the expression of ex, a well-characterized Hippo target gene, in imaginal discs. Clones with $\alpha$-spec RNAi showed a significant increase in Ex protein levels and a modest increase in ex transcription in the pupal retina (Figure 2A-C'). Similarly, $\alpha$-spec RNAi by the engrailed-Gal4 led to a significant increase in Ex protein and transcription levels (Figure 2-figure supplement $1 \mathrm{~A}-\mathrm{C}^{\prime \prime}$ ). Consistent with the upregulation of Hippo target genes, increased nuclear accumulation of Yki was observed in $\alpha$-spec ${ }^{\text {rg } 41}$ mutant cells (Figure 2D- $\left.D^{\prime \prime \prime}\right)$. We next examined the expression of diap1, another well-characterized Hippo target gene. Similar to mer or kibra mutant clones, which display no obvious increase in diap 1 expression (Pellock et al., 2007; Yu et al., 2010), clones with $\alpha$-spec RNAi showed no visible increase in Diap1 protein levels (Figure 2E-E'). Interestingly, combinations of $\alpha$-spec RNAi with any of these mutations ( $\alpha$-spec RNAi;kibradel or $\alpha$-spec RNAi;mer ${ }^{4}$ ) resulted a significant elevation in Diap1 protein levels (Figure $2 \mathrm{~F}-\mathrm{G}^{\prime}$ and Figure 2-figure supplement $\left.1 D-E^{\prime \prime}\right)$, further implicating spectrin as an upstream regulator of Hippo signaling. 


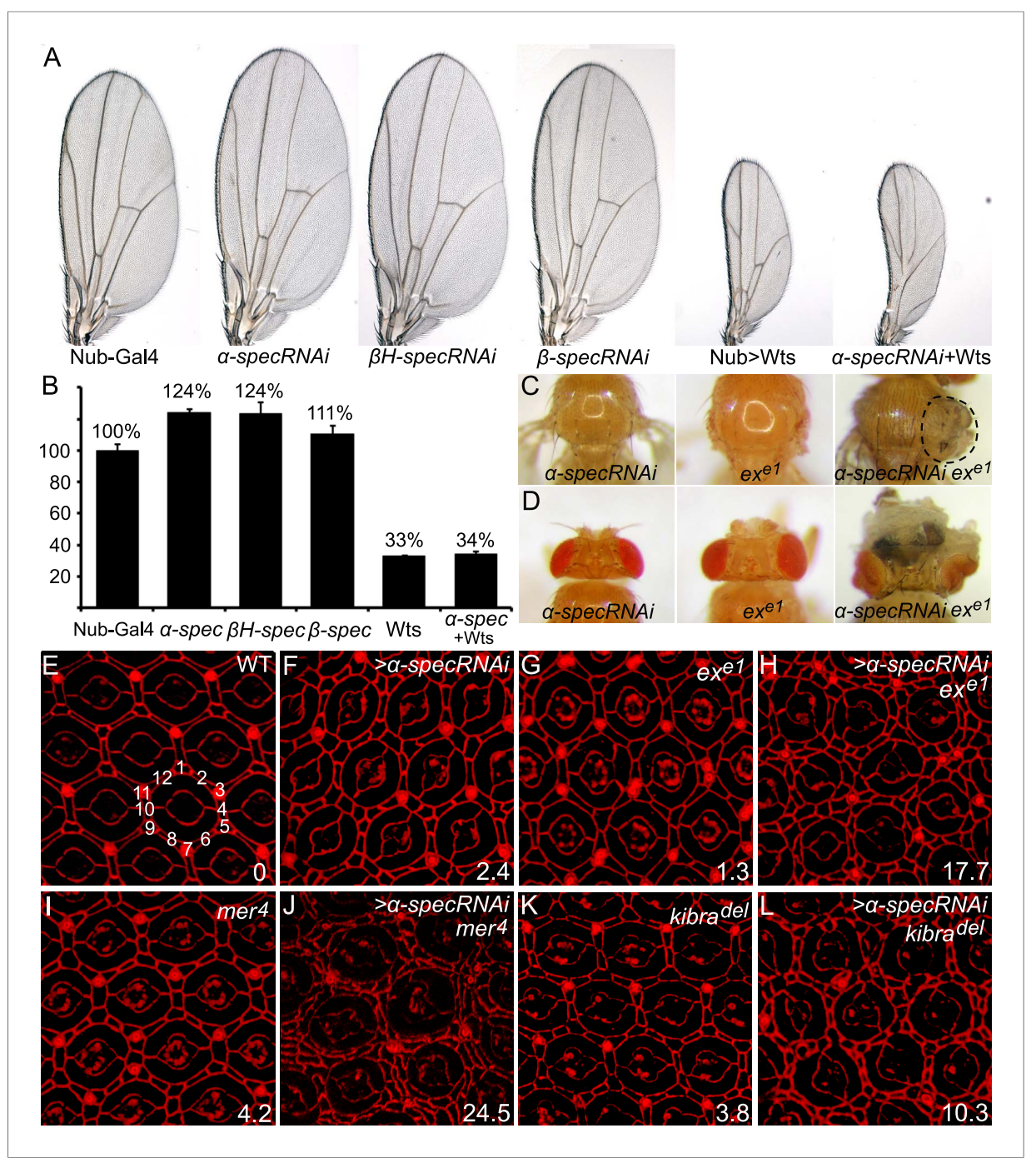

Figure 1. Spectrins function synergistically with upstream regulators of Hippo signaling to control tissue growth. (A-B) RNAi transgene against each of the three spectrin genes and UAS-wts were expressed separately or in combination in the wing tissue by nub-Gal4. Representative adult wings are shown. The graph in $\mathbf{B}$ shows quantification of wing size relative to nub-Gal4/+ control (mean $\pm \mathrm{SEM}, \mathrm{n}=15$ ). (C-D) MARCM clones with $\alpha$-spec RNAi, ex ${ }^{e 1}$ mutation or their combination were produced in the notum (C) or in the eye tissues (D). Note the massive overgrowth (circled area) only in flies containing ex mutant clones with $\alpha$-spec RNAi. (E-L) Pupal eye discs of the indicated genotypes were stained for DE-cad. Twenty ommatidial clusters of each genotype were used for counting interommatidial cells, and the number on the lower right of each panel indicates the number of extra cells per cluster (ECPC).

DOI: 10.7554/eLife.06567.003

The following figure supplements are available for figure 1:

Figure supplement 1. Analysis of $\alpha$-Spec and $\beta \mathrm{H}-\mathrm{Spec}$ localization and RNAi knockdown efficiency in imaginal disc epithelial cells

DOI: 10.7554/eLife.06567.004

Figure supplement 2. RNAi of $\beta \mathrm{H}$-spec or $\beta$-spec, or a null allele of $\alpha$-spec or $\beta$-spec, phenocopies the overgrowth phenotype of $\alpha$-spec RNAi in pupal retina.

DOI: 10.7554/eLife.06567.005

To test whether spectrin is a regulator of Hippo signaling beyond the imaginal discs, we examined the Drosophila ovary, where Hippo signaling is required in the posterior follicle cells (PFCs) for a mitosis-to-endoreplication switch between stages 6 and 7. Complete loss of Hippo signaling, as shown by mutants lacking components of the core kinase cascade, results in prolonged expression of 

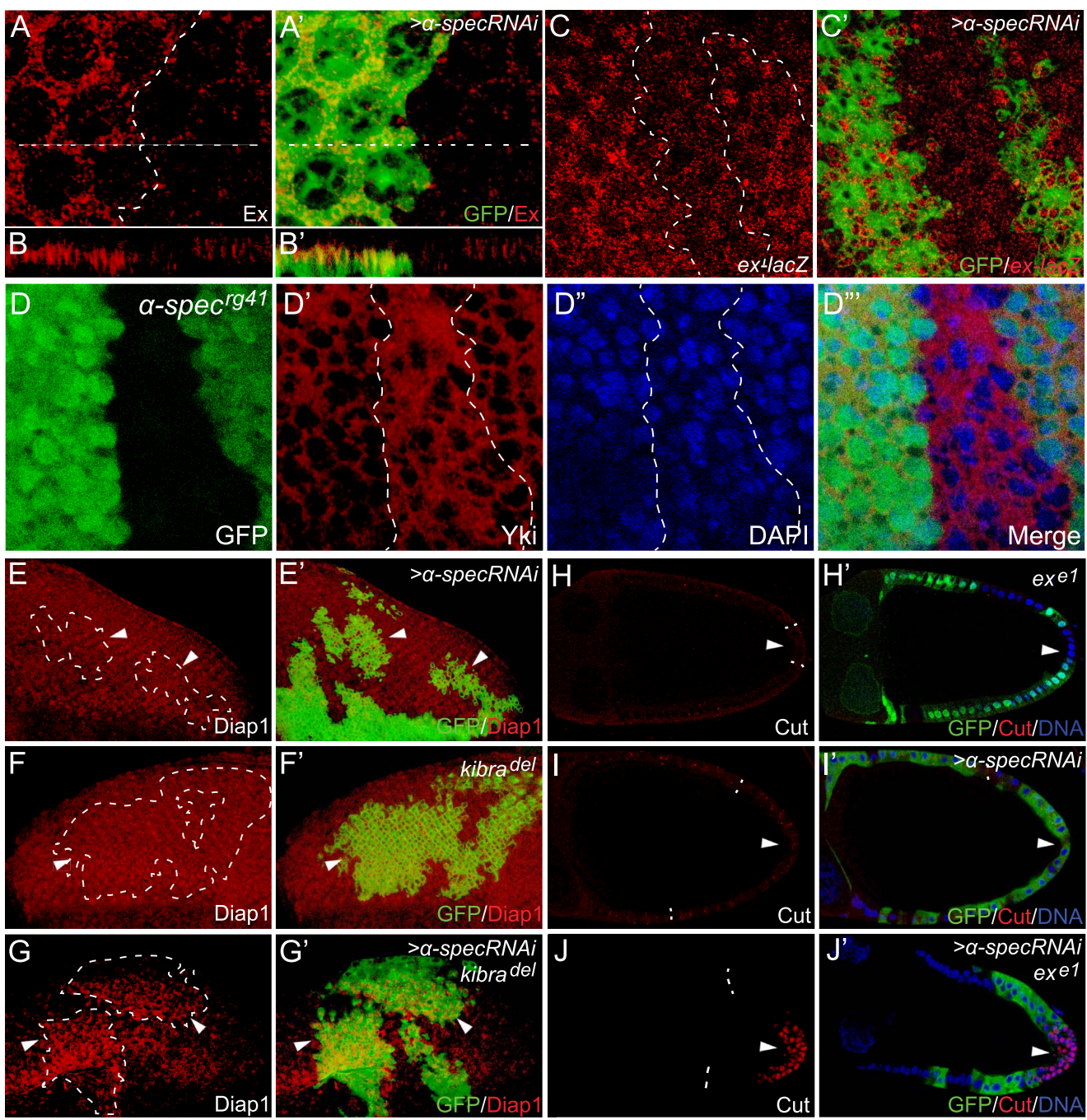

Figure 2. $\alpha$-Spec regulates the expression of Hippo target genes. (A-C') Pupal eye discs containing GFP-positive MARCM clones with $\alpha$-spec RNAi were stained for Ex (red, in $\mathbf{A}-\mathbf{B}^{\prime}$ ) or ex-lacZ (red, in $\mathbf{C}-\mathbf{C}^{\prime}$ ). B-B' shows a vertical section through the eye disc in $\mathbf{A}-\mathbf{A}^{\prime}$, in which the position of the vertical section is indicated by a straight dotted line. Note the elevated Ex level (A and B) or LacZ level (C) in clones with $\alpha$-spec RNAi. (D-D"') A third instar wing disc containing GFP-negative $\alpha$-spec ${ }^{r g 41}$ mutant clones was stained for Yki protein. Note the increased nuclear Yki signal in many $\alpha$-spec ${ }^{\text {rg } 41}$ mutant cells. (E-G') Third instar eye discs containing GFP-positive MARCM clones of the indicated genotypes were stained for Diap1 expression. Note the normal expression of Diap1 in clones with $\alpha$-spec RNAi $\left(\mathbf{E}-\mathbf{E}^{\prime}\right)$ or kibra ${ }^{\text {del }}$ mutant $\left(\mathbf{F}-\mathbf{F}^{\prime}\right)$, and the elevated Diap1 levels in kibra ${ }^{\text {del }}$ mutant clones with $\alpha$-spec RNAi (G-G'). (H-J') Stage 10 egg chambers containing GFP-negative ex ${ }^{e 1}$ mutant clones $\left(\mathbf{H}-\mathbf{H}^{\prime}\right)$, GFP-positive MARCM clones with $\alpha$-spec RNAi (I-I') or GFP-positive ex ${ }^{\mathrm{e} 1}$ mutant clones with $\alpha$-spec RNAi $\left(\mathbf{J}-\mathbf{J}^{\prime}\right)$ were stained for Cut expression. Cut expression and multilayering of follicle cells were observed only in the ex ${ }^{\mathrm{e}}$ mutant clones with $\alpha$-spec RNAi (J-J').

DOI: 10.7554/eLife.06567.006

The following figure supplements are available for figure 2 :

Figure supplement 1. $\alpha$-Spec regulates the expression of Hippo target genes. DOl: 10.7554/eLife.06567.007

Figure supplement 2. Loss of $\alpha$-Spec does not affect the subcellular localization of Mer or Kibra. DOI: 10.7554/eLife.06567.008 
cut and formation of multilayer PFCs in stage 10 egg chambers (Meignin et al., 2007; Polesello and Tapon, 2007; Yu et al., 2008). In contrast, inactivation of upstream regulators such as Ex leads to a milder phenotype characterized by transiently prolonged cut expression that disappears by stage 10 (Yu et al., 2008) (Figure 2H-H'). Similar to the ex mutation, $\alpha$-spec RNAi in PFCs did not cause visible upregulation of cut expression in stage 10 egg chambers (Figure 2l-l'). However, $\alpha$-spec RNAi in ex mutant clones produced multilayer of PFCs with strong cut expression persisted in stage 10 (Figure $\left.2 \mathrm{~J}-J^{\prime}\right)$. Thus, spectrin functions as a widespread regulator of Hippo signaling in multiple tissue contexts. The requirement of spectrin in both imaginal discs and PFCs distinguishes it from Ft-Ds, which is required in imaginal discs but dispensable in the PFCs (Bennett and Harvey, 2006; Cho et al., 2006; Silva et al., 2006; Willecke et al., 2006; Meignin et al., 2007; Polesello and Tapon, 2007; Yu et al., 2008).

Next, we investigated how spectrin regulates Hippo signaling. Since spectrin usually functions as a scaffold protein at the membrane-cytoskeleton interface, we first examined the subcellular localization of three membrane-associated upstream regulators of Hippo signaling: Ex, Mer, and Kibra. Of note, similar to spectrin, these proteins regulate Hippo signaling in both imaginal discs and PFCs (Hamaratoglu et al., 2006; Meignin et al., 2007; Polesello and Tapon, 2007; Yu et al., 2008; Baumgartner et al., 2010; Genevet et al., 2010; Yu et al., 2010). We detected no visible changes in the subcellular localization of these proteins (Figure 2A-B' and Figure 2-figure supplement 2). Together with the synergist effect of spectrin knockdown with ex, mer, or kibra mutations described above, these results suggest that spectrin likely functions in parallel with Ex, Mer, and Kibra to regulate Hippo signaling.

Given recent studies implicating the actomyosin cytoskeleton as a regulator of Hippo signaling, we examined the possibility that the SBMS may regulate Hippo signaling through the actomyosin cytoskeleton, whose major components are non-muscle myosin II and F-actin. As the major force generator in most cell types, the activity of non-muscle myosin II is regulated by phosphorylation of the regulatory light chain of myosin II (MLC) by multiple kinases including Rho-associated protein kinase (Rok) (Vicente-Manzanares et al., 2009). Phosphorylation of MLC greatly increases the $\mathrm{Mg}^{2+}$-ATPase activity of myosin in the presence of actin and leads to the generation of contractile forces or tension (Somlyo and Somlyo, 2003). Interestingly, a significant increase in p-MLC was observed in the eye imaginal disc upon RNAi knockdown of $\alpha$-spec, $\beta$-spec, or $\beta H$-spec (Figure 3). Careful examination of the $\mathrm{p}-\mathrm{MLC}$ signal revealed a polarized effect of spectrin knockdown that is consistent with the subcellular distribution of the different spectrin subunits (Lee et al., 1997) (see also Figure 1-figure supplement 1): reducing $\alpha$-Spec led to elevated p-MLC level in both apical and basolateral cortices of the cells (Figure $3 A-B^{\prime}$ ), but reducing $\beta \mathrm{H}-\mathrm{Spec}$ caused increased $\mathrm{p}-\mathrm{MLC}$ only in the apical cortices (Figure $3 C-D^{\prime}$ ) while reducing $\beta$-Spec caused increased $\mathrm{p}-\mathrm{MLC}$ only in the basolateral cortices (Figure $3 E-F^{\prime}$ ). A similar increase of p-MLC was observed in the wing imaginal disc upon $\alpha$-spec knockdown (Figure 3I-l') and confirmed in mutant clones carrying the $\alpha$-spec ${ }^{\text {rg } 41}$ allele (Figure $3 G-G^{\prime}$ ). Despite the increase in p-MLC level, the expression of MLC was unaffected in $\alpha$-spec ${ }^{\text {rg }} 41$ mutant clones, as measured by GFP staining from a genomic rescue spaghetti squash (sqh: encoding MLC in Drosophila)-GFP construct (Royou et al., 2004) (Figure 3H-H'), suggesting that the increased p-MLC level is not simply due to an overall increase in total MLC levels. Despite the changes of $\mathrm{p}-\mathrm{MLC}$ level, phalloidin staining did not reveal gross abnormality in the overall level and integrity of actin cytoskeleton in $\alpha$-spec ${ }^{\text {rg41 }}$ mutant cells (Figure $3 \mathrm{~J}-\mathrm{J}^{\prime}$ ). Of note, the regulation of p-MLC is specific to spectrin, as changes of p-MLC were not observed in mutant clones for other Hippo pathway regulators such as ex, mer, kibra, and crb, or mutant clones for the core kinase component wts (Figure $4 A-E^{\prime}$ ). These findings implicate spectrin as the only tumor suppressor identified to date that regulates Hippo signaling by modulating cortical actomyosin contractility.

To examine whether MLC activation contributes to the overgrowth phenotype produced by spectrin knockdown, we dampened MLC activation by expressing a rok RNAi transgene in mutant clones with $\alpha$-spec RNAi. Indeed, loss of Rok suppressed the clonal overgrowth of $\alpha$-spec mutant tissues (Figure 5A-D). Concurrent with the suppression of tissue overgrowth, loss of Rok also reversed the increased p-MLC level and the extra interommatidial cells seen in the $\alpha$-spec mutant tissues (Figure $\left.5 E-F^{\prime \prime \prime}\right)$. In fact, the $\alpha$-spec rok double RNAi clones resemble rok RNAi clones as both showed decreased growth, decreased levels of $\mathrm{p}-\mathrm{MLC}$ and decreased number of interommatidial cells

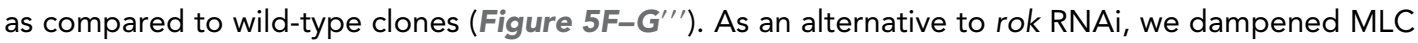
activation by expressing a dominant negative form of Rok (Rok ${ }^{\mathrm{KG}}$ ) (Winter et al., 2001) and observed 

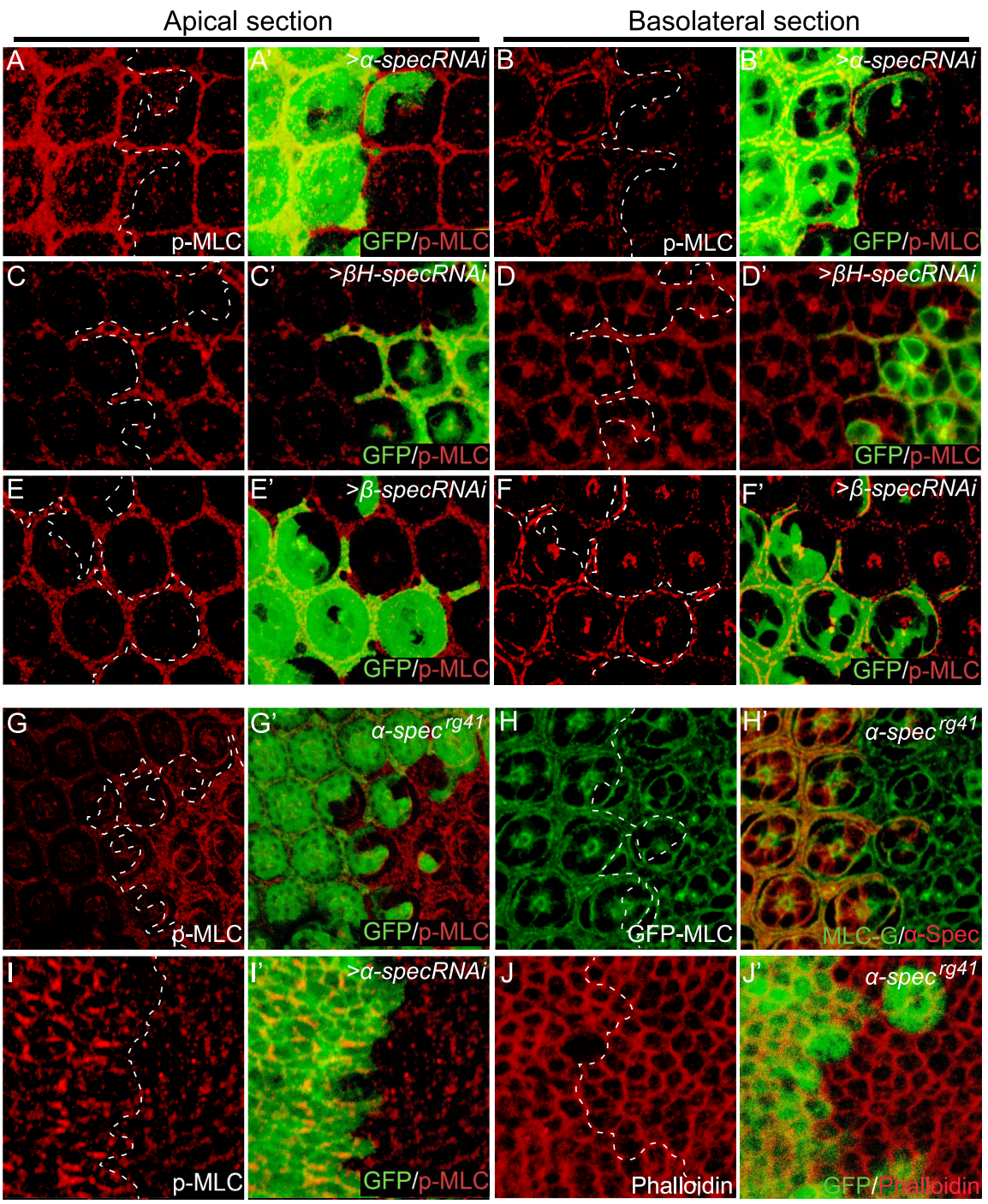

Figure 3. Loss of spectrin promotes the phosphorylation and activation of MLC. (A-F') Pupal eye discs containing GFP-positive MARCM clones with $\alpha$-spec, $\beta$ H-spec, or $\beta$-spec RNAi were stained for phospho-MLC (p-MLC, red). For each imaginal disc, both apical section $\left(\mathbf{A}-\mathbf{A}^{\prime}, \mathbf{C}-\mathbf{C}^{\prime}\right.$ and $\left.\mathbf{E}-\mathbf{E}^{\prime}\right)$ and basolateral confocal section (B-B', $\mathbf{D}-\mathbf{D}^{\prime}$ and $\left.\mathbf{F}-\mathbf{F}^{\prime}\right)$ were shown. Note the increase of $\mathrm{p}-\mathrm{MLC}$ in both apical and basolateral sections of the $\alpha$-spec mutant cells $\left(\mathbf{A}-\mathbf{A}^{\prime}\right.$ and $\left.\mathbf{B}-\mathbf{B}^{\prime}\right)$, the increase of $\mathrm{p}-\mathrm{MLC}$ only in the apical section of $\beta \mathrm{H}$-spec mutant cells $\left(\mathbf{C}-\mathbf{C}^{\prime}\right.$ and $\left.\mathbf{D}-\mathbf{D}^{\prime}\right)$, and the increase of $\mathrm{p}-\mathrm{MLC}$ only in the basolateral section of the $\beta$-spec mutant cells $\left(\mathbf{E}-\mathbf{E}^{\prime}\right.$ and $\left.\mathbf{F}-\mathbf{F}^{\prime}\right)$. (G-G') A pupal eye disc containing GFP-negative $\alpha$-spec ${ }^{r g 41}$ mutant clones stained for p-MLC. Note significant increase of p-MLC level in the mutant clone. $\left(\mathbf{H}-\mathbf{H}^{\prime}\right)$ A pupal eye disc containing GFP-negative $\alpha$-spec $c^{\text {rg41 }}$ mutant clones was stained for $\alpha$-Spec (red) and Sqh-GFP (green). Note the similar levels of Sqh-GFP expression inside and outside the $\alpha$-spec ${ }^{\text {rg }}{ }^{41}$ mutant clones. (I-I') A Third instar wing disc containing GFP-positive MARCM clones with $\alpha$-spec RNAi was stained for p-MLC. Note significant increase of p-MLC level in clones with $\alpha$-spec RNAi. (J-J') A Third instar wing disc containing GFP-negative $\alpha$-spec $c^{\text {r91 }}$ mutant clones was stained for F-actin using phalloidin (red). Note the similar actin cytoskeleton organization in $\alpha$-spec ${ }^{\text {rg41 }}$ mutant clones.

DOI: 10.7554/eLife.06567.009

a similar suppression of $\alpha$-spec mutant phenotypes (data not shown). These data are consistent with spectrin acting upstream of MLC to regulate Hippo signaling. In agreement with this genetic epistasis, overexpression of Wts in $\alpha$-spec mutant clones completely reversed the increased number of interommatidial cells without affecting the elevated p-MLC level in these clones (Figure $\left.4 F-G^{\prime \prime}\right)$. These data further support our model implicating spectrin as an upstream regulator of Hippo signaling. 

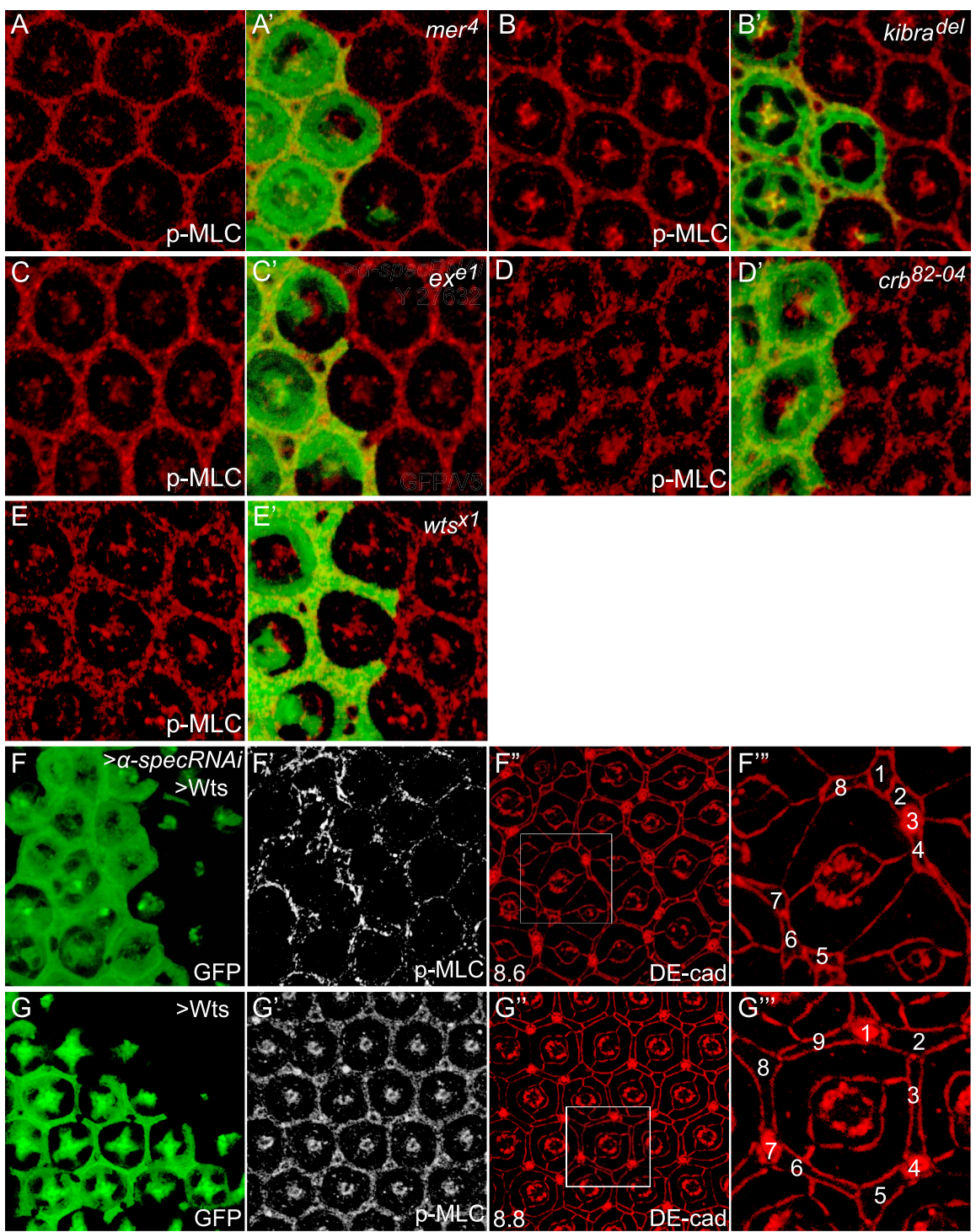

Figure 4. Canonical upstream tumor suppressors of the Hippo pathway do not regulate p-MLC activity. (A-E') Pupal eye discs containing GFP-positive MARCM clones of the indicated mutations were stained for P-MLC. Note the similar levels of $\mathrm{p}-\mathrm{MLC}$ inside and outside the mutant clones. (F-F"') A pupal eye disc containing GFP-positive MARCM clones with $\alpha$-spec RNAi and Wts overexpression, showing upregulation of $\mathrm{p}-\mathrm{MLC}\left(\mathbf{F}^{\prime}\right)$ and decreased number of interommatidial cells $\left(\mathbf{F}^{\prime \prime}\right.$; the number in the lower left represents the average number of interommatidial cells surrounding each unit eye calculated from 20 mutant ommatidia). The magnified view of a representative ommatidium in $\mathbf{F}^{\prime \prime}$ (boxed area) is shown in $\mathbf{F}^{\prime \prime \prime}$, with all the interommatidial cells marked by different numbers. Wild-type eyes have an average of 12 interommatidial cells surrounding each unit eye (Carthew, 2007) (see also Figure 1E). (G-G'") Similar to $\mathbf{F}-\mathbf{F}^{\prime \prime \prime}$ except that MARCM clones with $\mathbf{W t s}$ overexpression were analyzed. The magnified view of a representative ommatidium in $\mathbf{G}^{\prime \prime}$ (boxed area) is shown in $\mathbf{G}^{\prime \prime}$. Note the similar level of p-MLC $\left(\mathbf{G}^{\prime}\right)$ in the clones compared to the neighboring wild-type tissues. Also note the decreased interommatidial cell number compared to wild-type eyes. DOI: 10.7554/eLife.06567.010

It was recently reported that increased cytoskeleton tension suppresses Hippo signaling by recruiting the Ajuba (Jub)-Wts complex to the apical junctions (Rauskolb et al., 2014). To investigate whether spectrin regulates Hippo signaling through the Jub-Wts complex, we examine the subcellular localization of Jub and Wts in spectrin-defective cells using Wts-V5, Wts-GFP and Jub-GFP reporters (Rauskolb et al., 2014). Despite the increased actomyosin activity in $\alpha$-spec knockdown cells 

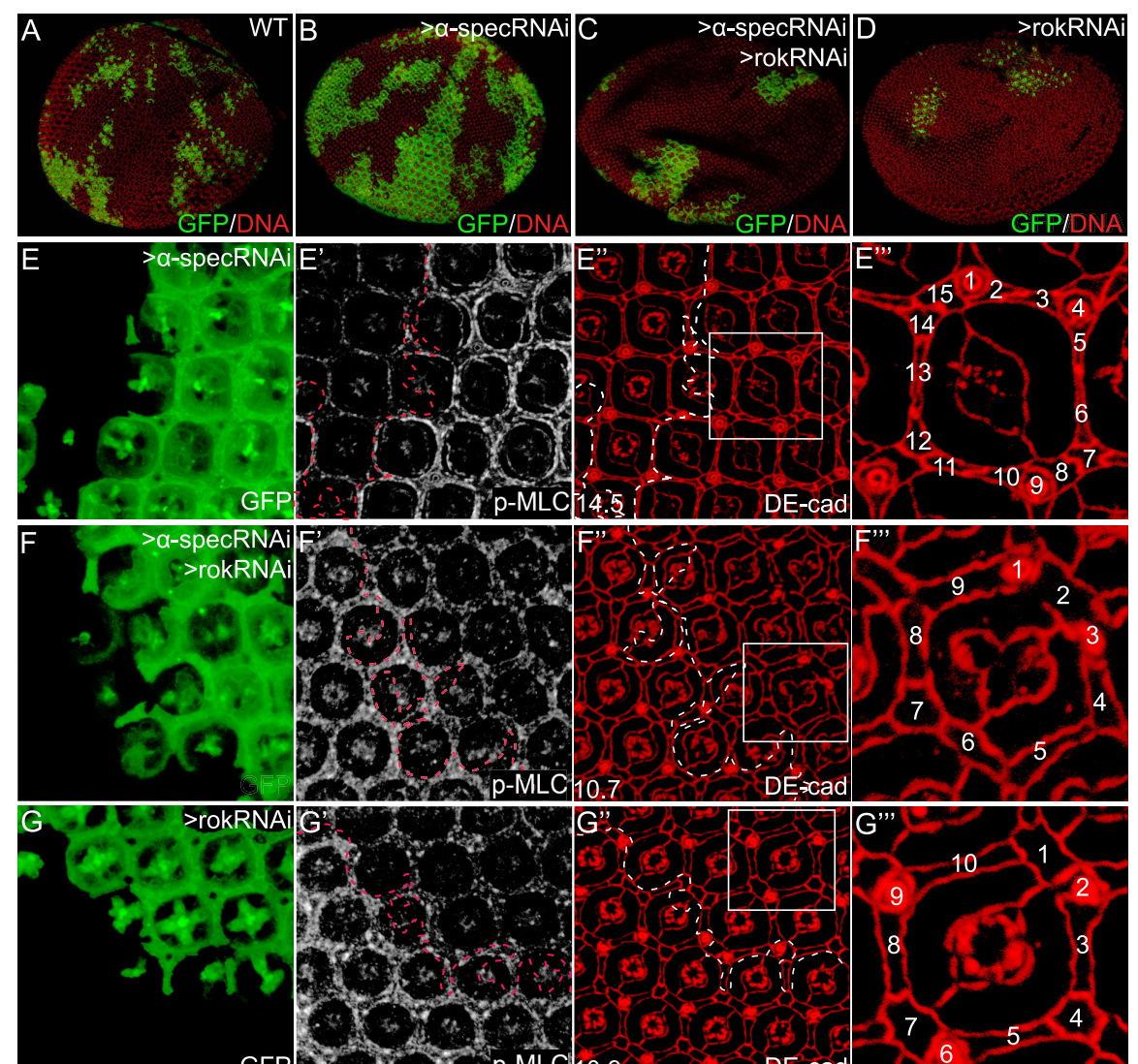

GFP

Figure 5. Inhibition of MLC activation suppresses the $\alpha$-spec-deficient phenotypes. (A-D) Pupal eye discs containing GFP-positive MARCM clones of the indicated genotypes. Note the increased clone size resulting from $\alpha$-spec RNAi (compare the relative representation of GFP-positive tissues in the whole eye between $\mathbf{B}$ and $\mathbf{A}$ ). Also note the decreased representation of the GFP-positive clones resulting from rok RNAi (D) or $\alpha$-spec rok RNAi (C). (E-E'") A pupal eye disc containing GFP-positive MARCM clones with $\alpha$-spec RNAi, showing upregulation of p-MLC (E') and increased number of interommatidial cells ( $\mathbf{E}^{\prime \prime}$; the number in the lower left represents the average number of interommatidial cells surrounding each unit eye calculated from 20 mutant ommatidia). The magnified view of a representative ommatidium in $\mathbf{E}^{\prime \prime}$ (boxed area) is shown in $\mathbf{E}^{\prime \prime \prime}$, with all the interommatidial cells marked by different numbers. (F-F"') Similar to $\mathbf{E}-\mathbf{E}^{\prime \prime \prime}$ except that MARCM clones with $\alpha$-spec and rok double RNAi were analyzed. Note the decrease of p-MLC $\left(\mathbf{F}^{\prime}\right)$ and interommatidial cell number $\left(\mathbf{F}^{\prime \prime}-\mathbf{F}^{\prime \prime}\right)$ in the clones compared to the neighboring wild-type tissues. Wild-type eyes have an average of 12 interommatidial cells surrounding each unit eye (Carthew, 2007) (see also Figure 1E). (G-G'") Similar to E-E"' except that MARCM clones with rok RNAi were analyzed. Note the decrease of $\mathrm{p}-\mathrm{MLC}\left(\mathbf{G}^{\prime}\right)$ and interommatidial cell number $\left(\mathbf{G}^{\prime \prime}-\mathbf{G}^{\prime \prime \prime}\right)$ in the clones compared to the neighboring wild-type tissues.

DOI: 10.7554/eLife.06567.011

The following figure supplement is available for figure 5 :

Figure supplement 1. Loss of $\alpha$-Spec does not affect the subcellular localization of Jub or Wts.

DOI: 10.7554/eLife.06567.012

(Figure 3I-I'), we did not observe detectable changes in subcellular localization of Wts or Jub proteins (Figure 5-figure supplement 1). These findings suggest that spectrin-regulated cortical actomyosin activity may regulate Hippo signaling through a different mechanism.

The above results suggest that the $\alpha$-spec mutant phenotypes are due to high actomyosin contractility in these cells. A prediction of this model is that elevating actomyosin activity may be sufficient to recapitulate these phenotypes. To test this hypothesis, we increased actomyosin activity directly by expressing an activated $\mathrm{MLC}\left(\mathrm{Sqh}^{\mathrm{EE}}\right)$, which mimics phosphorylated Sqh at T20 and S21 (Winter et al., 2001). The number of ECPC of ommatidium was used as a characteristic and 
semi-quantitative readout of defective Hippo signaling in the pupal retina (Figure 6). Consistent with the hypothesis, expression of Sqh ${ }^{\mathrm{EE}}$ produced 3.6 ECPC, similar to the mer mutants (Figure 6A-D). Moreover, expression of $\mathrm{Sqh}{ }^{\mathrm{EE}}$ in mer or ex mutant clones led to a dramatic increase in the number of ECPC (Figure 6E-F), suggesting a synergistic effect between cytoskeletal tension and the canonical upstream regulators of the Hippo pathway in tissue growth. This synergism was further confirmed by evaluating the expression of the Hippo target gene Diap1. While neither Sqh ${ }^{\mathrm{EE}}$-overexpressing clones nor $\mathrm{mer}^{4}$ mutant clones in the pupal retina showed upregulation of Diap1 expression, mer $^{4}$ mutant clones with Sqh ${ }^{\mathrm{EE}}$ overexpression showed a significant elevation of Diap1 level (Figure 6G-I'). These results further support the view that the spectrin and myosin II-mediated cytoskeletal tension functions in parallel with the canonical upstream regulators of the Hippo pathway to regulate downstream signaling.

Finally, we examined whether the spectrin may play a conserved role in regulating Hippo signaling in mammalian cells. The $\alpha$ spectrin subunit is encoded by two genes in humans: SPTA1 which is expressed mainly in erythrocytes, and SPTAN1 which is expressed in all non-erythrocyte cells (Moon and McMahon, 1990; Cianci et al., 1999; Berghs et al., 2000). When the expression of SPTAN1 was knocked down through small interfering RNA (siRNA) in MCF10A cells (a human mammary epithelial cell line), we observed increased nuclear localization of YAP compared to control cells in confluent cultures (Figure 7A-B'"), as well as increased cortical p-MLC level (Figure 7C-D'). Consistent with immunostaining, SPTAN1 RNAi led to decreased YAP phosphorylation at the Hippo-responsive Ser 127 and Ser 381 sites compared to the control cells, and also increased p-MLC (Figure 7E). These results suggest that the SBMS may play a conserved role in regulating actomyosin activity and Hippo signaling in mammalian cells.

\section{Discussion}

Although the actomyosin-mediated cytoskeletal tension has been implicated as a regulator of Hippo signaling from insects to mammalian cells, how the actomyosin cytoskeleton itself is modulated remains largely unknown. Through the identification of all the spectrin subunits as negative growth regulators (tumor suppressors) and the characterization of their mechanism of action in Drosophila, we have uncovered the SBMS, a cytoskeleton directly underneath the plasma membrane, as an essential regulator of the actomyosin cytoskeleton in Hippo signaling. The negative regulation of myosin II activity is unique to the spectrin, since it is not shared by other known upstream tumor suppressors of the Hippo pathway. Rather, the spectrin-Myo II pathway functions in parallel with the other upstream regulators to modulate Hippo signaling in vivo. Although spectrin was reported to regulate the Hippo pathway during the preparation and review of this paper (Fletcher et al., 2015; Wong et al., 2015), our study is the first to uncover a functional link between the SBMC and Myo II activity, or to establish the parallel relationship between spectrin-Myoll-mediated actomyosin activity and the other known upstream regulators of Hippo signaling. To our knowledge, this is the first demonstration that a structural membrane skeleton protein regulates Hippo signaling by modulating actomyosin contractility.

Our study also provides new insight into the nature of force production that is relevant to Hippo signaling. Previous studies implicating cytoskeletal tension as a regulator of Hippo signaling were based on whole-cell perturbation of the actomyosin cytoskeleton (Dupont et al., 2011; Wada et al., 2011; Aragona et al., 2013; Rauskolb et al., 2014). Since the actomyosin cytoskeleton is distributed in diverse subcellular compartments such as the cell cortex, the cytoplasm and the cell-cell junction, it was unclear from these experiments which subcellular pool(s) of the actomyosin cytoskeleton are relevant to Hippo signaling. Our observation that loss of the SBMS leads to defective Hippo signaling with elevated $\mathrm{p}-\mathrm{MLC}$ levels only around the cell cortex suggests that the actomyosin cytoskeleton directly underneath the plasma membrane is functionally linked to the Hippo pathway. Interestingly, ablation of apical spectrin ( $\beta \mathrm{H}$-spec) and basolateral spectrin ( $\beta$-spec) leads to localized activation of $\mathrm{p}-\mathrm{MLC}$ in the apical and basolateral cortex, respectively. Yet, both ablations result in similar Hippo-related growth defects. Based on these findings, we infer that the actomyosin cytoskeleton in both membrane domains is relevant to Hippo signaling, in contrast to a previous report suggesting that only apical actin polymerization affects Hippo signaling (Fernandez et al., 2011).

While our study has uncovered a functional link between spectrin and actomyosin activity, the molecular mechanisms by which actomyosin activity regulates Hippo signaling remain to be determined. At present, there are contrasting views on whether cytoskeleton-mediated regulation of Yki/YAP activity 

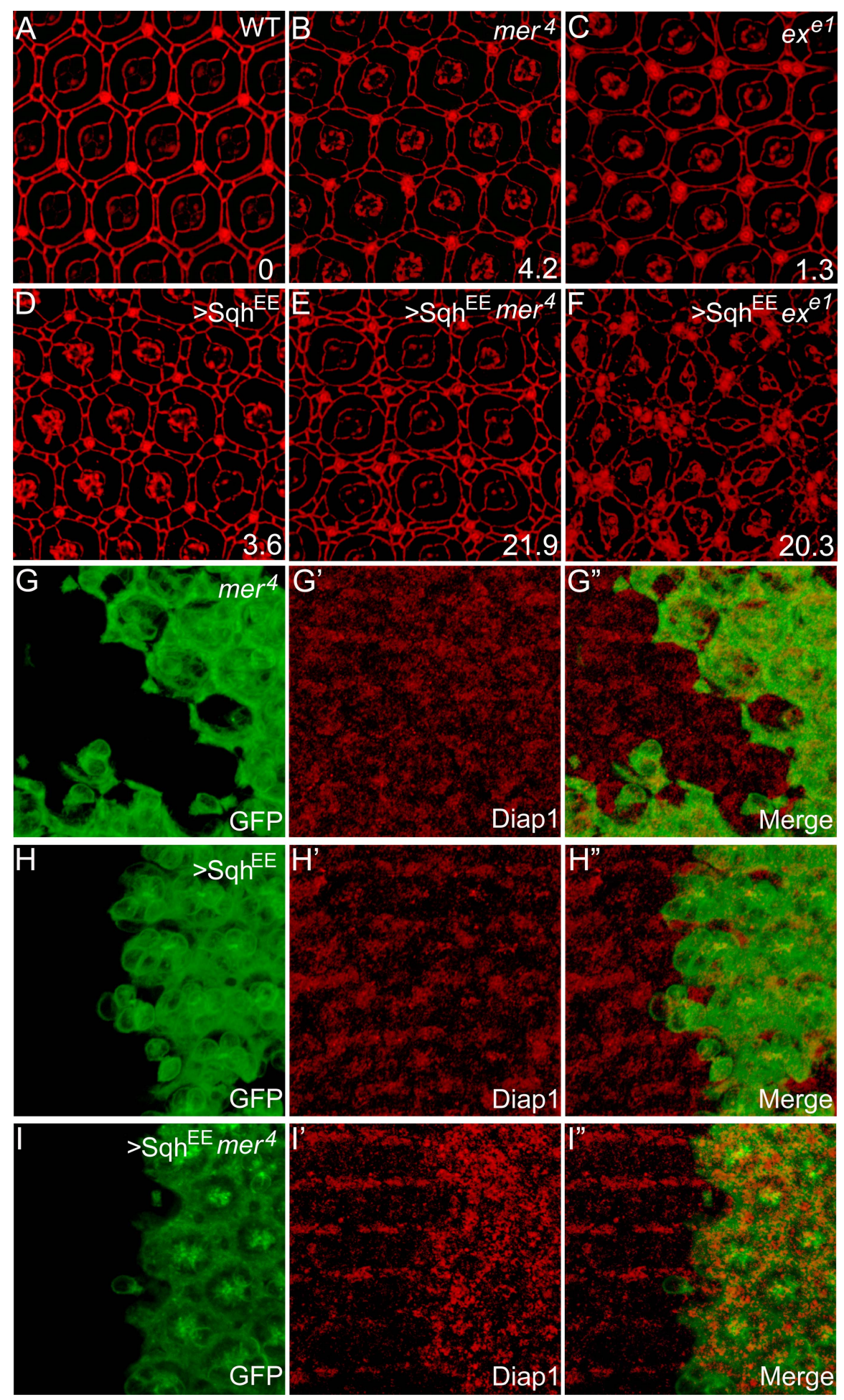

Figure 6. Myosin II-regulated cytoskeletal tension functions in parallel with the canonical upstream tumor suppressors to regulate Hippo signaling. (A-F) The synergistic effect of myosin II activation and loss of Ex or Mer on interommatidial cell number. Pupal eye discs of the indicated genotype were stained for DE-cad. 20 ommatidial clusters of each genotype were used for counting interommatidial cells, and the number on the lower right of each panel indicates the average number of ECPC. Note the dramatic increase of ECPC in $\mathbf{E}$ and $\mathbf{F}$. (G-I') The synergistic effect of myosin II activation and loss of Mer on Hippo target gene expression. Pupal eye discs containing Figure 6. continued on next page 
Figure 6. Continued

GFP-positive MARCM clones of the indicated genotypes were stained for Diap1 expression. Note the normal expression of Diap1 in mer ${ }^{4}$ mutant clones $\left(\mathbf{G}^{\prime}\right)$ or Sqh ${ }^{\mathrm{EE}}$-overexpressing clones $\left(\mathbf{H}^{\prime}\right)$, and the elevated Diap1 levels in $\mathrm{mer}^{4}$ mutant clones with Sqh${ }^{\mathrm{EE}}$ overexpression $\left(\mathbf{I}^{\prime}\right)$.

DOI: 10.7554/eLife.06567.013

is Wts/Lats-dependent or Wts/Lats-independent (reviewed in Low et al., 2014). Our characterization of spectrin is more consistent with a Wts/LATS-dependent mechanism, since YAP phosphorylation is decreased by spectrin knockdown in mammalian cells (Figure 7E) and that overexpression of Wts can rescue the ECPC phenotype of spectrin RNAi without rescuing the high p-MLC levels in Drosophila (Figure 4F). Nevertheless, our data cannot exclude the possibility of Wts/Lats-independent mechanisms.

Previous studies have focused on the different functions of the SBMS and the actomyosin cytoskeleton in cell biology. Much of the studies on SBMS have emphasized its role in the
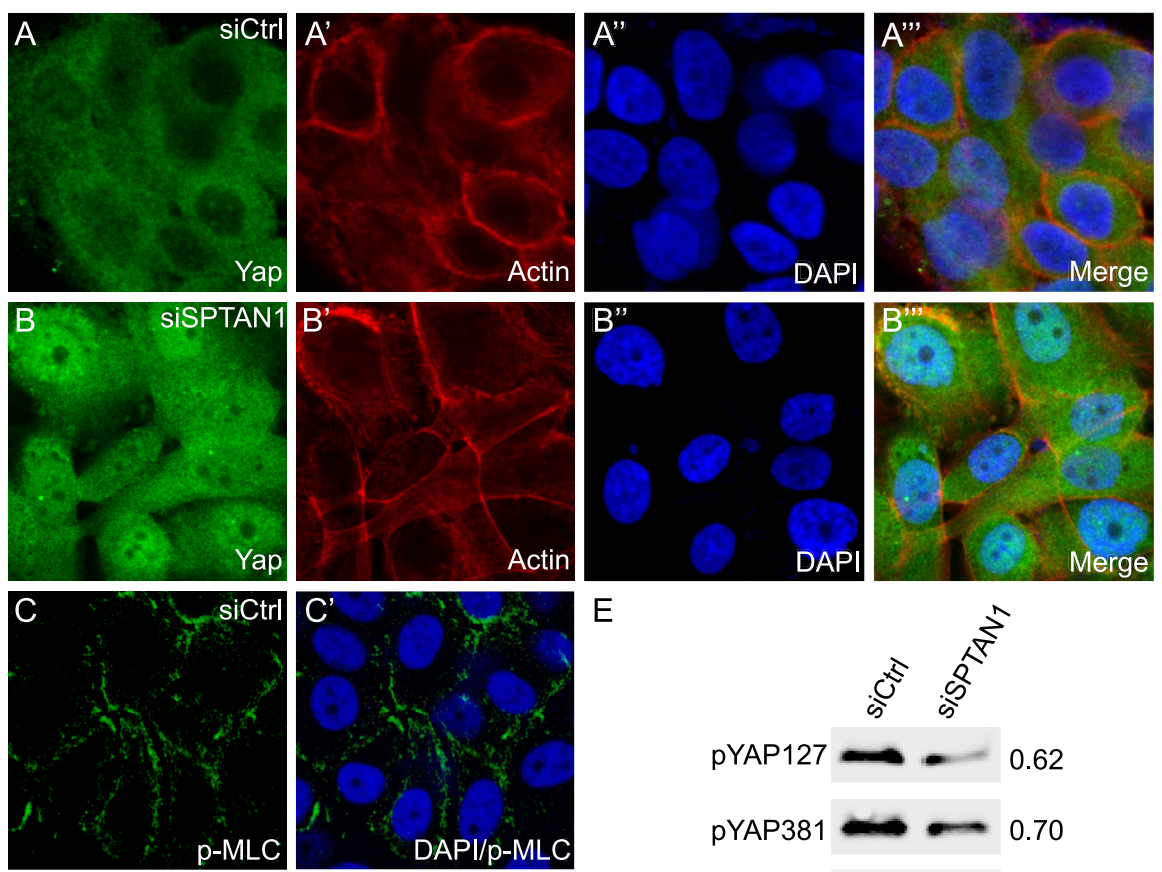

$\mathrm{E}$
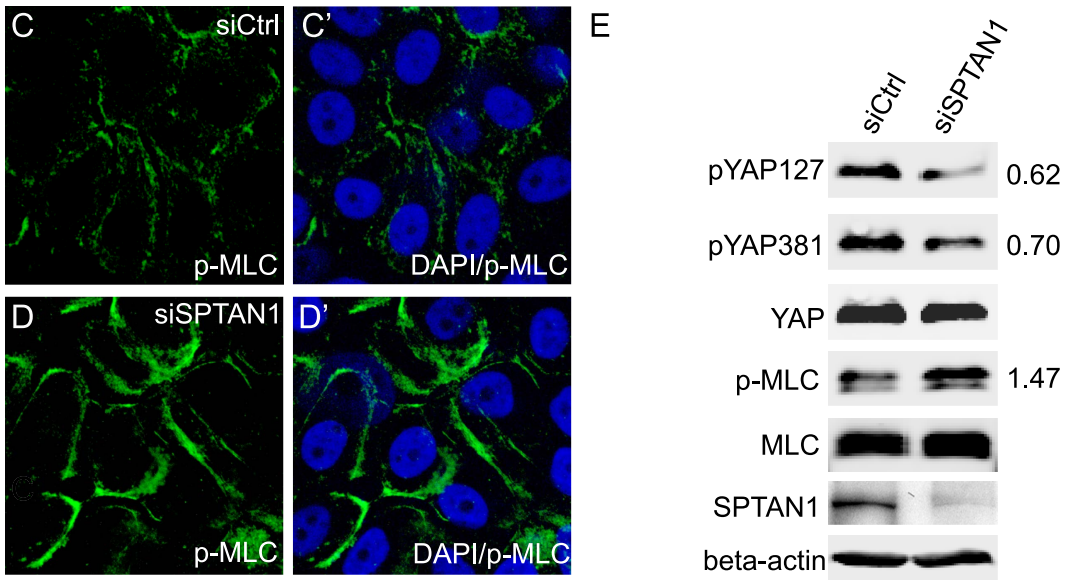

Figure 7. Loss of SPTAN1 results in decreased YAP phosphorylation, increased YAP nuclear localization, and increased cortical p-MLC level in MCF10A cells. (A-B"') Confluent cultures of MCF10A cells treated with control RNAi or SPTAN1 RNAi were stained for YAP (green), actin (red) and the nuclear dye DAPI (blue). Note the increased nuclear YAP signal in cells with SPTAN1 RNAi. (C-D') Confluent cultures of MCF10A cells treated with control RNAi or SPTAN1 RNAi were stained for p-MLC (green) and DAPI (blue). Note the increased cortical p-MLC signal in cells with SPTAN1 RNAi. (E) Western blot analysis of cells from $\mathbf{A}-\mathbf{D}^{\prime}$. Quantification of $\mathrm{p}$-YAP to total YAP ratio (or p-MLC to total MLC ratio) in the SPTAN1 RNAi cells, normalized to that in the control RNAi cells, is shown to the right. Note the decreased YAP S127 and S381 phosphorylation and increased p-MLC level in cells with SPTAN1 RNAi.

DOI: 10.7554/eLife.06567.014 
maintenance of plasma membrane integrity, while studies of the actomyosin cytoskeleton have implicated it in diverse cellular processes from mechanotransduction to cell migration (Bennett and Baines, 2001; Lecuit and Lenne, 2007; Campellone and Welch, 2010; Lecuit et al., 2011; Machnicka et al., 2012; Takeichi, 2014). Although spectrin subunits have been reported to physically interact with several proteins that may directly or indirectly impact the actomyosin cytoskeleton, such as F-actin, Adducin, $\alpha$-Catenin and Moesin (Gardner and Bennett, 1987; Pradhan et al., 2001; Medina et al., 2002; Barkalow et al., 2003; Xu et al., 2013), functional coupling between these two cytoskeleton systems remains poorly understood. Our current study uncovers a previously underappreciated functional link between these two cytoskeleton systems and demonstrates that this link is physiologically important for regulating the Hippo pathway in multiple developmental contexts. We speculate that mechanical stimuli on cell membrane may be transduced first to the SBMS directly underneath the plasma membrane, which then regulates actomyosin tension to influence cell behaviors. Conversely, the actomyosin cytoskeleton may also regulate the function of the SBMS (Fukata et al., 1999). Understanding the detailed molecular mechanism by which the SBMS regulates Myo II activity will provide an important entry point to dissect the crosstalk between the SBMS and the actomyosin cytoskeleton in diverse cellular processes.

\section{Materials and methods}

\section{Drosophila genetics}

UAS- $\alpha$-specRNAi, UAS- $\beta$-specRNAi and UAS- $\beta H$-specRNAi lines were obtained from Vienna Drosophila Resource Center (VDRC, stock ID 25387, 42054 and 37075). UAS-rokRNAi was obtained from the Bloomington Drosophila Stock Center (stock ID 34324). The following flies have been described previously: $\alpha$-spec ${ }^{r g 41}$ (Lee et al., 1993), $\beta$-spec ${ }^{C}$ (Yamamoto et al., 2014), ex ${ }^{11}$ (Boedigheimer and Laughon, 1993), $\operatorname{mer}^{4}$ (Fehon et al., 1997), kibradel (Yu et al., 2010), crb ${ }^{82-04}$ (Ling et al., 2010), wts $^{X 1}$ (Xu et al., 1995), UAS-Wts (Yin et al., 2013), Sqh-GFP (Royou et al., 2004), UAS-Sqh ${ }^{\mathrm{EE}}$ and UAS-Rok ${ }^{\mathrm{KG}}$ (Winter et al., 2001), Wts-V5, Wts-GFP and Jub-GFP (Rauskolb et al., 2014). All crosses were done at $25^{\circ} \mathrm{C}$. The following genotypes were used for clonal analysis: Control MARCM clones:

UAS-GFP hs-flp; tub-Gal80 FRT40A/FRT40A; tub-Gal4/+

MARCM clones with RNAi of $\alpha$-spec, $\beta$-spec, $\beta \mathrm{H}$-spec or rok:

UAS-GFP hs-flp; tub-Gal80 FRT40A/FRT40A UAS- $\alpha$-specRNAi; tub-Gal4/+ UAS-GFP hs-flp; tub-Gal80 FRT40A/FRT40A UAS- $\beta$-specRNAi; tub-Gal4/+ 19A /19A tub-Gal80 hs-flp; UAS- $\beta$ H-specRNAi / UAS-GFP; tub-Gal4/+ UAS-GFP hs-flp; tub-Gal80 FRT40A/FRT40A; tub-Gal4/UAS-rokRNAi

MARCM clones expressing constitutively active MLC (Sqh $\left.{ }^{\mathrm{EE}}\right)$ :

UAS-GFP hs-flp; tub-Gal80 FRT40A/FRT40A UAS-sgh ${ }^{\mathrm{EE}}$; tub-Gal4/+

MARCM clones with $\alpha$-spec and rok double RNAi:

UAS-GFP hs-flp; tub-Gal80 FRT40A/FRT40A UAS- $\alpha$-specRNAi; tub-Gal4/UAS-rokRNAi

MARCM clones with both $\alpha$-spec RNAi and Sqh ${ }^{\mathrm{EE}}$ overexpression:

UAS-GFP hs-flp; tub-Gal80 FRT40A/FRT40A UAS- $\alpha$-specRNAi, UAS- sqh ${ }^{\mathrm{EE}}$; tub-Gal4/+

mer, ex, kibra, crb or wts mutant MARCM clones:

19A mer 4 /19A tub-Gal80 hs-flp; UAS-GFP/+; tub-Gal4/+ UAS-GFP hs-flp; tub-Gal80 FRT40A/FRT40A ex ${ }^{\mathrm{e} 1}$; tub-Gal4/+ UAS-GFP hs-flp; tub-Gal4/+; tub-Gal80 FRT82B/FRT82B kibra del UAS-GFP hs-flp; tub-Gal4/+; tub-Gal80 FRT82B/FRT82B crb $^{82-04}$ UAS-GFP hs-flp; tub-Gal4/+; tub-Gal80 FRT82B/FRT82B wts ${ }^{\times 1}$

mer, ex or kibra mutant MARCM clones with $\alpha$-spec RNAi:

19A mer 4 /19A tub-Gal80 hs-flp; UAS-GFP/UAS- $\alpha$-specRNAi; tub-Gal4/+ UAS-GFP hs-flp; tub-Gal80 FRT40A/FRT40A ex ${ }^{\mathrm{e}}$, UAS- $\alpha$-specRNAi; tub-Gal4/+ UAS-GFP hs-flp; tub-Gal4/ UAS- $\alpha$-specRNAi; tub-Gal80 FRT82B/FRT82B kibradel 


\section{Cell culture, siRNA transfection, and immunofluorescence staining}

MCF10A cells were cultured in DMEM/F12 (Invitrogen, Carlsbad, California) supplemented with 5\% horse serum, $20 \mathrm{ng} / \mathrm{ml} \mathrm{EGF,} 0.5 \mu \mathrm{g} / \mathrm{ml}$ hydrocortisone, $10 \mu \mathrm{g} / \mathrm{ml}$ insulin, $100 \mathrm{ng} / \mathrm{ml}$ cholera toxin, and $50 \mu \mathrm{g} / \mathrm{ml}$ penicillin/streptomycin. Cells were maintained in a $37^{\circ} \mathrm{C}$ incubator at $5 \% \mathrm{CO}_{2}$. Cells were cultured on Lab-Tek II Chamber Slide (Thermo Scientific, Waltham, Massachusetts) to $80-85 \%$ confluent, and then siRNA transfections were performed twice using Lipofectamine RNAiMAX Reagent with a 24-hr interval. Cells were processed for immunoblotting or immunofluorescence $96 \mathrm{hr}$ post-transfection. SMARTpool siRNA oligonucleotides toward human SPTAN1 and Non-Targeting siRNA Pool \#2 (control siRNA) were purchased from GE Dharmacon (Lafayette, Colorado). For immunostaining, cells were fixed and stained following standard method using anti-YAP antibody (Novus, 1:300 dilution) and anti-p-MLC antibody (1:10, Cell Signaling Technologies, Beverly, Massachusetts). Western blotting was done using antibodies against following proteins: pYAP127 (1:1000, Cell Signaling Technologies); pYAP381 (1:1000, Cell Signaling Technologies); YAP (1:200, Sigma, St. Louis, Missouri); MLC (1:1000, Cell Signaling Technologies); SPTAN1 (1: 500, Santa Cruz Biotechnology, Dallas, Texas); and beta-actin (1:200, Sigma).

\section{Acknowledgements}

We thank Dr Rick Fehon for Ex and Mer antibodies, Dr Nic Tapon for Kibra antibody, and the Bloomington and Vienna Stock Centers for Drosophila stocks. This work was supported in part by grants from the National Institutes of Health (EY015708). DP is an investigator of the Howard Hughes Medical Institute.

\section{Additional information}

Competing interests

DP: Reviewing editor, eLife. The other authors declare that no competing interests exist.

Funding

\begin{tabular}{lll} 
Funder & Grant reference & Author \\
\hline $\begin{array}{l}\text { Howard Hughes Medical } \\
\text { Institute (HHMI) }\end{array}$ & Investigator & Duojia Pan \\
$\begin{array}{lll}\text { National Institutes of Health } \\
\text { (NIH) }\end{array}$ & EY015708 & $\begin{array}{l}\text { Hua Deng, Wei Wang, } \\
\text { Jianzhong Yu, Yonggang } \\
\text { Zheng, Yun Qing, Duojia Pan }\end{array}$ \\
\hline
\end{tabular}

The funders had no role in study design, data collection and interpretation, or the decision to submit the work for publication.

Author contributions

HD, Conception and design, Acquisition of data, Analysis and interpretation of data, Drafting or revising the article; WW, JY, YZ, YQ, Conception and design, Acquisition of data, Analysis and interpretation of data; DP, Conception and design, Analysis and interpretation of data, Drafting or revising the article

\section{References}

Aragona M, Panciera T, Manfrin A, Giulitti S, Michielin F, Elvassore N, Dupont S, Piccolo S. 2013. A mechanical checkpoint controls multicellular growth through YAP/TAZ regulation by actin-processing factors. Cell 154: 1047-1059. doi: 10.1016/j.cell.2013.07.042.

Baines AJ. 2009. Evolution of spectrin function in cytoskeletal and membrane networks. Biochemical Society Transactions 37:796-803. doi: 10.1042/BST0370796.

Barkalow KL, Italiano JE Jr, Chou DE, Matsuoka Y, Bennett V, Hartwig JH. 2003. Alpha-adducin dissociates from F-actin and spectrin during platelet activation. The Journal of Cell Biology 161:557-570. doi: 10.1083/jcb. 200211122.

Barry ER, Camargo FD. 2013. The Hippo superhighway: signaling crossroads converging on the Hippo/Yap pathway in stem cells and development. Current Opinion in Cell Biology 25:247-253. doi: 10.1016/j.ceb.2012.12.006.

Baumgartner R, Poernbacher I, Buser N, Hafen E, Stocker H. 2010. The WW domain protein Kibra acts upstream of hippo in Drosophila. Developmental Cell 18:309-316. doi: 10.1016/j.devcel.2009.12.013. 
Bennett FC, Harvey KF. 2006. Fat cadherin modulates organ size in Drosophila via the Salvador/Warts/Hippo signaling pathway. Current Biology 16:2101-2110. doi: 10.1016/j.cub.2006.09.045.

Bennett V, Baines AJ. 2001. Spectrin and ankyrin-based pathways: metazoan inventions for integrating cells into tissues. Physiological Reviews 81:1353-1392.

Bennett V, Gilligan DM. 1993. The spectrin-based membrane skeleton and micron-scale organization of the plasma membrane. Annual Review of Cell and Developmental Biology 9:27-66. doi: 10.1146/annurev.cb.09. 110193.000331.

Berghs S, Aggujaro D, Dirkx R Jr, Maksimova E, Stabach P, Hermel JM, Zhang JP, Philbrick W, Slepnev V, Ort T, Solimena M. 2000. betalV spectrin, a new spectrin localized at axon initial segments and nodes of ranvier in the central and peripheral nervous system. The Journal of Cell Biology 151:985-1002. doi: 10.1083/jcb.151.5.985.

Boedigheimer M, Laughon A. 1993. Expanded: a gene involved in the control of cell proliferation in imaginal discs. Development 118:1291-1301.

Boggiano JC, Fehon RG. 2012. Growth control by committee: intercellular junctions, cell polarity, and the cytoskeleton regulate hippo signaling. Developmental Cell 22:695-702. doi: 10.1016/j.devcel.2012.03.013.

Campellone KG, Welch MD. 2010. A nucleator arms race: cellular control of actin assembly. Nature Reviews Molecular Cell Biology 11:237-251. doi: 10.1038/nrm2867.

Carthew RW. 2007. Pattern formation in the Drosophila eye. Current Opinion in Genetics \& Development 17: 309-313. doi: 10.1016/j.gde.2007.05.001.

Cho E, Feng Y, Rauskolb C, Maitra S, Fehon R, Irvine KD. 2006. Delineation of a Fat tumor suppressor pathway. Nature Genetics 38:1142-1150. doi: 10.1038/ng1887.

Cianci CD, Zhang Z, Pradhan D, Morrow JS. 1999. Brain and muscle express a unique alternative transcript of alphall spectrin. Biochemistry 38:15721-15730. doi: 10.1021/bi991458k.

Delaunay J. 2007. The molecular basis of hereditary red cell membrane disorders. Blood Reviews 21:1-20. doi: 10. 1016/j.blre.2006.03.005.

Dupont S, Morsut L, Aragona M, Enzo E, Giulitti S, Cordenonsi M, Zanconato F, Le DJ, Forcato M, Bicciato S, Elvassore N, Piccolo S. 2011. Role of YAP/TAZ in mechanotransduction. Nature 474:179-183. doi: 10.1038/ nature10137.

Enderle L, McNeill H. 2013. Hippo gains weight: added insights and complexity to pathway control. Science Signaling 6:re7. doi: 10.1126/scisignal.2004208.

Fehon RG, Oren T, LaJeunesse DR, Melby TE, McCartney BM. 1997. Isolation of mutations in the Drosophila homologues of the human Neurofibromatosis 2 and yeast CDC42 genes using a simple and efficient reversegenetic method. Genetics 146:245-252.

Fernandez BG, Gaspar P, Bras-Pereira C, Jezowska B, Rebelo SR, Janody F. 2011. Actin-Capping Protein and the Hippo pathway regulate F-actin and tissue growth in Drosophila. Development 138:2337-2346. doi: 10.1242/ dev.063545.

Fletcher GC, Elbediwy A, Khanal I, Ribeiro PS, Tapon N, Thompson BJ. 2015. The Spectrin cytoskeleton regulates the Hippo signalling pathway. The EMBO Journal 34:940-954. doi: 10.15252/embj.201489642.

Fukata Y, Oshiro N, Kinoshita N, Kawano Y, Matsuoka Y, Bennett V, Matsuura Y, Kaibuchi K. 1999. Phosphorylation of adducin by Rho-kinase plays a crucial role in cell motility. The Journal of Cell Biology 145:347-361. doi: 10. 1083/jcb.145.2.347.

Gardner K, Bennett V. 1987. Modulation of spectrin-actin assembly by erythrocyte adducin. Nature 328:359-362. doi: 10.1038/328359a0.

Genevet A, Wehr MC, Brain R, Thompson BJ, Tapon N. 2010. Kibra is a regulator of the Salvador/Warts/Hippo signaling network. Developmental Cell 18:300-308. doi: 10.1016/j.devcel.2009.12.011.

Halder G, Johnson RL. 2011. Hippo signaling: growth control and beyond. Development 138:9-22. doi: 10.1242/ dev.045500.

Hamaratoglu F, Willecke M, Kango-Singh M, Nolo R, Hyun E, Tao C, Jafar-Nejad H, Halder G. 2006. The tumoursuppressor genes NF2/Merlin and expanded act through Hippo signalling to regulate cell proliferation and apoptosis. Nature Cell Biology 8:27-36. doi: 10.1038/ncb1339.

Harvey K, Tapon N. 2007. The Salvador-Warts-Hippo pathway-an emerging tumour-suppressor network. Nature Reviews Cancer 7:182-191. doi: 10.1038/nrc2070.

Harvey KF, Zhang X, Thomas DM. 2013. The Hippo pathway and human cancer. Nature Reviews Cancer 13: 246-257. doi: 10.1038/nrc3458.

Johnson R, Halder G. 2014. The two faces of Hippo: targeting the Hippo pathway for regenerative medicine and cancer treatment. Nature Reviews Drug Discovery 13:63-79. doi: 10.1038/nrd4161.

Krieg M, Dunn AR, Goodman MB. 2014. Mechanical control of the sense of touch by beta-spectrin. Nature Cell Biology 16:224-233. doi: 10.1038/ncb2915.

Lecuit T, Lenne PF. 2007. Cell surface mechanics and the control of cell shape, tissue patterns and morphogenesis. Nature Reviews Molecular Cell Biology 8:633-644. doi: 10.1038/nrm2222.

Lecuit T, Lenne PF, Munro E. 2011. Force generation, transmission, and integration during cell and tissue morphogenesis. Annual Review of Cell and Developmental Biology 27:157-184. doi: 10.1146/annurev-cellbio100109-104027.

Lee JK, Brandin E, Branton D, Goldstein LS. 1997. alpha-Spectrin is required for ovarian follicle monolayer integrity in Drosophila melanogaster. Development 124:353-362.

Lee JK, Coyne RS, Dubreuil RR, Goldstein LS, Branton D. 1993. Cell shape and interaction defects in alpha-spectrin mutants of Drosophila melanogaster. The Journal of Cell Biology 123:1797-1809. doi: 10.1083/jcb.123.6.1797. 
Ling C, Zheng Y, Yin F, Yu J, Huang J, Hong Y, Wu S, Pan D. 2010. The apical transmembrane protein Crumbs functions as a tumor suppressor that regulates Hippo signaling by binding to expanded. Proceedings of the National Academy of Sciences of USA 107:10532-10537. doi: 10.1073/pnas.1004279107.

Low BC, Pan CQ, Shivashankar GV, Bershadsky A, Sudol M, Sheetz M. 2014. YAP/TAZ as mechanosensors and mechanotransducers in regulating organ size and tumor growth. FEBS Letters 588:2663-2670. doi: 10.1016/j. febslet.2014.04.012.

Machnicka B, Grochowalska R, Boguslawska DM, Sikorski AF, Lecomte MC. 2012. Spectrin-based skeleton as an actor in cell signaling. Cellular and Molecular Life Sciences 69:191-201. doi: 10.1007/s00018-011-0804-5.

Medina E, Williams J, Klipfell E, Zarnescu D, Thomas G, Le Bivic A. 2002. Crumbs interacts with moesin and beta (Heavy)-spectrin in the apical membrane skeleton of Drosophila. The Journal of Cell Biology 158:941-951. doi: 10.1083/jcb.200203080.

Meignin C, Alvarez-Garcia I, Davis I, Palacios IM. 2007. The salvador-warts-hippo pathway is required for epithelial proliferation and axis specification in Drosophila. Current Biology 17:1871-1878. doi: 10.1016/j.cub.2007.09.062.

Moon RT, McMahon AP. 1990. Generation of diversity in nonerythroid spectrins. Multiple polypeptides are predicted by sequence analysis of cDNAs encompassing the coding region of human nonerythroid alphaspectrin. The Journal of Biological Chemistry 265:4427-4433.

Pan D. 2007. Hippo signaling in organ size control. Genes \& Development 21:886-897. doi: 10.1101/gad.1536007.

Pan D. 2010. The hippo signaling pathway in development and cancer. Developmental Cell 19:491-505. doi: 10. 1016/j.devcel.2010.09.011.

Pellock BJ, Buff E, White K, Hariharan IK. 2007. The Drosophila tumor suppressors expanded and Merlin differentially regulate cell cycle exit, apoptosis, and Wingless signaling. Developmental Biology 304:102-115. doi: 10.1016/j.ydbio.2006.12.021.

Polesello C, Tapon N. 2007. Salvador-warts-hippo signaling promotes Drosophila posterior follicle cell maturation downstream of notch. Current Biology 17:1864-1870. doi: 10.1016/j.cub.2007.09.049.

Pradhan D, Lombardo CR, Roe S, Rimm DL, Morrow JS. 2001. alpha -Catenin binds directly to spectrin and facilitates spectrin-membrane assembly in vivo. The Journal of Biological Chemistry 276:4175-4181. doi: 10 1074/jbc.M009259200.

Rauskolb C, Sun S, Sun G, Pan Y, Irvine KD. 2014. Cytoskeletal tension inhibits Hippo signaling through an AjubaWarts complex. Cell 158:143-156. doi: 10.1016/j.cell.2014.05.035.

Royou A, Field C, Sisson JC, Sullivan W, Karess R. 2004. Reassessing the role and dynamics of nonmuscle myosin II during furrow formation in early Drosophila embryos. Molecular Biology of the Cell 15:838-850. doi: 10.1091/ mbc.E03-06-0440.

Sansores-Garcia L, Bossuyt W, Wada K, Yonemura S, Tao C, Sasaki H, Halder G. 2011. Modulating F-actin organization induces organ growth by affecting the Hippo pathway. The EMBO Journal 30:2325-2335. doi: 10 . 1038/emboj.2011.157.

Silva E, Tsatskis Y, Gardano L, Tapon N, McNeill H. 2006. The tumor-suppressor gene fat controls tissue growth upstream of expanded in the hippo signaling pathway. Current Biology 16:2081-2089. doi: 10.1016/j.cub.2006. 09.004.

Somlyo AP, Somlyo AV. 2003. Ca2+ sensitivity of smooth muscle and nonmuscle myosin II: modulated by G proteins, kinases, and myosin phosphatase. Physiological Reviews 83:1325-1358. doi: 10.1152/physrev.00023. 2003.

Takeichi M. 2014. Dynamic contacts: rearranging adherens junctions to drive epithelial remodelling. Nature Reviews Molecular Cell Biology 15:397-410. doi: 10.1038/nrm3802.

Vicente-Manzanares M, Ma X, Adelstein RS, Horwitz AR. 2009. Non-muscle myosin II takes centre stage in cell adhesion and migration. Nature Reviews Molecular Cell Biology 10:778-790. doi: 10.1038/nrm2786.

Wada K, Itoga K, Okano T, Yonemura S, Sasaki H. 2011. Hippo pathway regulation by cell morphology and stress fibers. Development 138:3907-3914. doi: 10.1242/dev.070987.

Willecke M, Hamaratoglu F, Kango-Singh M, Udan R, Chen CL, Tao C, Zhang X, Halder G. 2006. The fat cadherin acts through the hippo tumor-suppressor pathway to regulate tissue size. Current Biology 16:2090-2100. doi: 10. 1016/j.cub.2006.09.005.

Winter CG, Wang B, Ballew A, Royou A, Karess R, Axelrod JD, Luo L. 2001. Drosophila Rho-associated kinase (Drok) links Frizzled-mediated planar cell polarity signaling to the actin cytoskeleton. Cell 105:81-91. doi: 10 1016/S0092-8674(01)00298-7.

Wong KK, Li W, An Y, Duan Y, Li Z, Kang Y, Yan Y. 2015. Beta-spectrin regulates the hippo signaling pathway and modulates the basal actin network. The Journal of Biological Chemistry 290:6397-6407. doi: 10.1074/jbc.M114. 629493.

Xu K, Zhong G, Zhuang X. 2013. Actin, spectrin, and associated proteins form a periodic cytoskeletal structure in axons. Science 339:452-456. doi: 10.1126/science.1232251.

Xu T, Wang W, Zhang S, Stewart RA, Yu W. 1995. Identifying tumor suppressors in genetic mosaics: the Drosophila lats gene encodes a putative protein kinase. Development 121:1053-1063.

Yamamoto S, Jaiswal M, Charng WL, Gambin T, Karaca E, Mirzaa G, Wiszniewski W, Sandoval H, Haelterman NA, Xiong B, Zhang K, Bayat V, David G, Li T, Chen K, Gala U, Harel T, Pehlivan D, Penney S, Vissers LE, de Ligt J, Jhangiani SN, Xie Y, Tsang SH, Parman Y, Sivaci M, Battaloglu E, Muzny D, Wan YW, Liu Z, Lin-Moore AT, Clark RD, Curry CJ, Link N, Schulze KL, Boerwinkle E, Dobyns WB, Allikmets R, Gibbs RA, Chen R, Lupski JR, Wangler MF, Bellen HJ. 2014. A Drosophila genetic resource of mutants to study mechanisms underlying human genetic diseases. Cell 159:200-214. doi: 10.1016/j.cell.2014.09.002. 
Yin F, Yu J, Zheng Y, Chen Q, Zhang N, Pan D. 2013. Spatial organization of hippo signaling at the plasma membrane mediated by the tumor suppressor Merlin/NF2. Cell 154:1342-1355. doi: 10.1016/j.cell.2013.08.025. Yu FX, Guan KL. 2013. The Hippo pathway: regulators and regulations. Genes \& Development 27:355-371. doi: 10.1101/gad.210773.112.

Yu J, Poulton J, Huang YC, Deng WM. 2008. The hippo pathway promotes Notch signaling in regulation of cell differentiation, proliferation, and oocyte polarity. PLOS ONE 3:e1761. doi: 10.1371/journal.pone.0001761.

Yu J, Zheng Y, Dong J, Klusza S, Deng WM, Pan D. 2010. Kibra functions as a tumor suppressor protein that regulates hippo signaling in conjunction with Merlin and expanded. Developmental Cell 18:288-299. doi: 10. 1016/j.devcel.2009.12.012.

Zhao B, Lei QY, Guan KL. 2008. The Hippo-YAP pathway: new connections between regulation of organ size and cancer. Current Opinion in Cell Biology 20:638-646. doi: 10.1016/j.ceb.2008.10.001. 